\title{
A importância da atividade física e alimentação na hipertensão arterial
}

DOI: $10.46919 / \operatorname{arch} 2 \mathrm{n} 2-003$

Recebimento dos originais: 01/01/2021

Aceitação para publicação: 31/03/2021

\section{Geovanna Porto Inácio}

Médica - Instituição de atuação atual: Instituto de Neurologia de Goiânia Endereço completo: Rua T-28, 1477, Setor Bueno. Goiânia - GO

E-mail: amgeovannapi@gmail.com

\section{Ana Paula Fontana}

Mestre em Ciências Ambientais e Saúde - Universidade de Rio Verde Endereço Completo: Rua Filhinho Portilho quadra 7 lote dois - Solar dos Ataídes I.

Rio Verde-Goiás

E-mail: fontana@unirv.edu.br

\section{Débora Bernardes Peixoto}

Médica residente em Clínica Médica - Hospital Regional de Sobradinho Endereço completo: SHIN CA5, Cj D3, Edifício Varandas do Lago Norte 1, Brasília- DF

E-mail: debora.bernardesp@hotmail.com

\section{Ariane Velasco Machado}

Médica - Instituição: Hospital e Maternidade Municipal Professora Maria Arlinda da Costa Endereço completo: Rua das Dálias, 34, Bairro Jardim Cuiabá, Cuiabá/MT E-mail: v.ariane.m@hotmail.com

\section{Fabrício De Souza Xavier}

Médico residente em Clínica Médica - Hospital Augusto de Oliveira Camargo (HAOC) Indaiatuba-SP Avenida Oliveira Morais Resende Quadra E Lote12, bairro Jardim Vale do Sol Uruana-GO

E-mail: fabricioxavier77@ @otmail.com

\section{Marina Santos Menezes}

Ensino Superior Incompleto - Medicina Instituição de Ensino: ITPAC - Porto Nacional Endereço completo: Rua Erasmo Braga, número 127. Centro. Anápolis- Goiás

E-mail: marinasantosm06@gmail.com

\section{Marcelo Albuquerque Rocha Aquino}

Ensino Superior Incompleto - Medicina Instituição de Ensino: ITPAC - Porto Nacional

SQS 212 B1 E APTO 204 - CEP 70.275-050 - Brasília-DF

E-mail: marceloaquino.med@gmail.com

\section{Jordanna Porto Inácio}

Ensino Superior Incompleto - Medicina Instituição de ensino: Faculdades Atenas

Sete Lagoas - Minas Gerais

Endereço completo: Rua T-28, 1477, Setor Bueno. Goiânia - GO

E-mail: jordannaporto@ hotmail.com 


\section{INTRODUÇÃO}

A hipertensão arterial sistêmica (HAS) é o maior fator de risco para mortalidade no mundo e transformou-se progressivamente em dos mais graves problemas de saúde pública. Em 2018, estimava-se que $12,8 \%$ das mortes foram decorrentes da HAS. Associada às várias diversidades sua prevalência sofre influência de múltiplos fatores, com destaque para os demográficos, hereditários, socioeconômicos, comportamentais e antropométricos. A maioria desses fatores podem ser controlado ou modificado, sendo então possível reduzir a incidência da hipertensão e de suas complicações. Além do que contribuiu nas últimas décadas para o aumento das cardiopatias, dos acidentes cerebrovasculares, a insuficiência renal e para as incapacidades prematuras (WHO, 2008; OLIVEIRA et al 2012; OMS 2013; MARQUES et al 2020; BARROSO et al 2021; OLIVEIRA et al 2021).

As evidências científicas apontaram que nos últimos anos as condutas não medicamentosas, como adesão aos programas de atividades físicas e alimentação adequada, constituem-se em estratégias iniciais na hipertensão leve a moderada. São ações de baixo custo e risco mínimo, capazes de contribuir para a regulação da pressão arterial, tratamento de dislipidemias e da obesidade. Na revisão sistemática, Marques et al (2020) referem que dentre os fatores de risco, a baixa atividade física representou maior chance de um indivíduo apresentar HAS (TAYLOR, 2014; CASSIANO et al 2020).

As Diretrizes Brasileiras para Hipertensão Arterial 2020 citam que há uma associação direta do sedentarismo com a elevação da PA e da HA. A falta de atividade física a maior prevalência estava entre as mulheres $(31,7 \%)$ do que nos homens $(23,4 \%)$. O que foi identificado em 2019 pelo inquérito telefônico Vigitel, onde 44,8\% dos adultos não alcançaram um nível suficiente de prática de atividade física, sendo esse percentual maior entre mulheres $(52,2 \%)$ em relação aos homens $(36,1 \%)$ (PICCINI et al 2012).

Existem muitas possibilidades de associar as atividades físicas a uma dieta equilibrada para prevenir e controlar a HA, sejam elas de baixa quantidade de gordura, mediterrânea, vegetariana, baixo teor de carboidratos e outras, além de incluir a baixa ingestão de sódio. No entanto, o maior desafio seja considerar e ser levados em conta os aspectos e as dificuldades socioeconômicos e culturais de cada população. Cassiano et al (2020) concluíram e recomendaram no seu estudo que um programa prolongado de exercícios físicos, associado ao acompanhamento nutricional possibilitará a redução do risco para o desenvolvimento de eventos cardiovasculares nos próximos dez anos, além de favorecer uma melhor qualidade de vida (OLIVEIRA et al 2012; ZAAR et al 2014; BRICARELLO et al 2020).

\section{OBJETIVO}

A partir de tal realidade, o objetivo dos acadêmicos de medicina de uma universidade privada foi realizar um evento destinado a conscientização da população em relação à prevenção e aos fatores de risco para a HAS. 


\section{METODOLOGIA}

Trata-se de um relato de experiência de caráter descritivo. Realizado por acadêmicos e professores da disciplina Medicina Integrada a Saúde da Comunidade da Faculdade de Medicina de Rio Verde, em Goiás. Foram realizadas atividades informativas e recreativas, com a intenção de esclarecimento de dúvidas sobre HAS, orientação de dieta equilibrada com base nas condições econômicas da população local, além de incentivar a prática de atividades física e distribuição de cartilhas explicativas sobre o tema.

Na experiência utilizou-se a problematização do Método do Arco de Charles Maguerez, apresentado pela primeira vez por Bordenave e Pereira, em 1982. É uma metodologia da Problematização utilizada em situações nas quais os temas estejam relacionados com a vida em sociedade. Assim, a partir da realidade social, são destacados em 5 etapas: a observação, os pontos-chave, a teorização, as hipóteses de solução e a aplicação à realidade. Cada tema é transformado em um problema para ser discutido em grupo. Os temas de estudo o acadêmico deverá saber e dominar, e que são determinados previamente com o seu professor e quais conhecimentos o acadêmico deverá possuir para cada um (ROCHA, 2008; PRADO et al 2012; CASSIANO et al 2020).

Foram coletados os dados como: idade, sexo, etnia, escolaridade, padrão alimentar, atividade física, se tabagista e/ou etilista. E a aferição da pressão arterial, de acordo com as Diretrizes Brasileiras de Hipertensão Arterial 2020 da Sociedade Brasileira de Cardiologia de PA < 140/90 mmHg (hipertensos em geral) ou < 130/80 mmHg (alto risco), mas atenção para os casos < 120/70 mmHg devido aos efeitos colaterais da hipotensão diastólica. A avaliação do Índice de Massa Corporal (sendo o parâmetro de normalidade $18,5-24,9 \mathrm{~kg} / \mathrm{m}$, acima desta medida correlacionado a sobrepeso e a obesidade, com grau de 29 risco) (Diretrizes Brasileiras de Obesidade, 2009/2010; Diretrizes Brasileiras para Hipertensão Arterial, 2020).

\section{RELATO DA EXPERIÊNCIA}

Durante a realização do evento observou-se que as pessoas participavam e demonstravam interesse pelos assuntos abordados, como certo contentamento pela oportunidade de aprendizagem ao expressarem a ajudarem nas atividades propostas. Para a equipe de saúde, principalmente para os acadêmicos de medicina essa experiência foi muito importante ao perceberem que a maioria dos pacientes hipertensos foram alertados por apresentarem vários fatores de risco, como: pressão alta, tabagismo, glicemia elevada, inatividade física e obesidade.

Nos resultados dos dados sobre a população participante, a maioria apresentaram com hipertensão arterial, eram do sexo feminino, assemelhando-se ao estudo de Piccini et al (2012), que foi realizado em 100 municípios do Brasil que constatou uma maior proporção de portadores de HAS em mulheres $(69,0 \%)$ do que em homens $(61,5 \%)$. 
Um alerta para a realização de atividades físicas, também se mostrou importante, já que dentre os hipertensos que participaram, a maioria não realizava qualquer tipo de atividade física. Dado encontrado de acordo com o estudo Taylor (2014), no qual demonstrou que a hipertensão e altas taxas de glicose no sangue, assim como obesidade estão relacionadas com a inatividade física.

Por meio das palestras ministradas e as informações repassadas durante o evento, os participantes poderão perceber que alterar hábitos, anteriormente equivocados em relação à sua alimentação diária e práticas de atividades físicas, podem prevenir, estabilizar e até mesmo reverter os quadros hipertensivos. Essa experiência, de ação implementada junto à comunidade trouxeram esperanças de mudança no estilo de vida dessas pessoas, pois vários participantes relataram que a partir daquele momento iriam tentar modificar o seu estilo de vida.

Um desses relatos foi de uma senhora que morava próximo a uma praça, sua alimentação continha muito sódio e que com esse encontro pode perceber que poderia utilizar da praça para fazer caminhadas e que com a sua persistência diminuiria o sódio de sua alimentação. Para os acadêmicos observarem que o simples fato de ouvir o paciente, valorizar o que ele é, e não somente a doença, que podem proporcionar melhor qualidade de vida e humanização da saúde fazem parte de gratidão à participação de toda comunidade assistida.

\section{DISCUSSÃO}

Houve o grande desafio de mostrar para a comunidade participante que existem várias maneiras não farmacológicas de prevenir e controlar a hipertensão, que se incluírem melhora dietética, atividade física e ajuste de peso, de mudanças do estilo de vida, consequentemente serão mais saudáveis. Importante destacar que para melhor adesão das pessoas, recomenda-se programas alimentares, orientações flexíveis e acessíveis aos hábitos, cultura e ao estilo de vida da população, com objetivos claros, direcionados para mudanças graduais, e com o acompanhamento de equipe multiprofissional de saúde.

\section{CONCLUSÃO}

Espera-se que os resultados dessa experiência contribuam para o debate e mais ações agregadas às comunidades sobre os fatores associados à HAS, apesar de serem multidimensionais, a conscientização da comunidade faz a diferença.

Palavras chave: atividade física, sedentarismo, obesidade, dieta, hipertensão arterial 


\section{REFERÊNCIAS}

ASSOCIAÇÃO BRASILEIRA PARA O ESTUDO DA OBESIDADE E DA SÍNDROME METABÓLICA. Diretrizes brasileiras de obesidade 2009/2010. 3.ed. - Itapevi, SP: AC Farmacêutica, 2009.

BARROSO, WKS et al. Diretrizes Brasileiras de Hipertensão Arterial - 2020. Arq. Bras. Cardiol., v. 116, n. 3, p. 516-658, mar. 2021.

BRICARELLO LP et al Abordagem dietética para controle da hipertensão: reflexões sobre adesão e possíveis impactos para a saúde coletiva. Ciência \& Saúde Coletiva, 25(4):1421-1432, 2020

CASSIANO AN et al. Efeitos do exercício físico sobre o risco cardiovascular e qualidade de vida em idosos hipertensos. Ciência \& Saúde Coletiva, 25(6):2203-2212, 2020

MARQUES AP et al. Fatores associados à hipertensão arterial: uma revisão sistemática. Ciência \& Saúde Coletiva, 25(6): 2271-2282, 2020

OLIVEIRA, E. P. et al. A variedade da dieta é fator protetor para a pressão arterial sistólica elevada. Arquivos Brasileiros de Cardiologia, vol.98, n.4, . 338-343, 2012.

ORGANIZACIÓN MUNDIAL DE LA SALUD (OMS). Información general sobre la hipertensión en el mundo. Una enfermedad que mata en silencio, una crisis de salud pública mundial. Día Mundial de la Salud 2013. Ginebra: OMS; 2013.

PRADO ML, VELHO MB, ESPÍNDOLA DS, SOBRINHO SH, BACKES VMS. Arco de Charles Maguerez: Refletindo Estratégias de Metodologia Ativa na Formação de Profissionais de Saúde. Esc Anna Nery (impr.)2012 jan-mar; 16 (1):172-177

PICCINI, R. X. et al. Promoção, prevenção e cuidado da hipertensão arterial no Brasil. Revista Saúde Pública, vol. 46, n.3, p.543-50, 2012.

ROCHA, R. O Método da Problematização: Prevenção às Drogas na Escola e o Combate a Violência. (Programa de Desenvolvimento Educacional da Secretaria Estadual de Educação) - Universidade Estadual de Londrina, 2008.

TAYLOR, D. Physical activity is medicine for older adults. Postgrad Medical Journal, vol. 90, n.10, p.26-32, 2014.

WORLD Health Organization (WHO). Global health risks: mortality and burden of disease attributable to selected major risks. Geneva: WHO; 2009.

ZAAR A, REIS VM, SBARDELOTTO ML. Efeitos de um programa de exercícios físicos sobre a pressão arterial e medidas antropométricas. Rev Bras Med Esporte 2014; 20(1):13-16. 